\title{
Etude expérimentale d'un déchargeur électrostatique pour hélicoptère
}

\author{
S. Larigaldie et N. Félici $(*)$ \\ Office National d'Etudes et de Recherches Aérospatiales, 92320 Châtillon, France \\ $\left({ }^{*}\right)$ Laboratoire d'Electrostatique, CNRS, 38000 Grenoble, France
}

(Reçu le 16 mai 1979, révisé le 8 octobre 1979, accepté le 10 octobre 1979)

\begin{abstract}
Résumé. - Le problème de l'éjection d'un courant de charges unipolaires d'un corps isolé est abordé. Un dispositif utilisant la condensation de l'humidité de l'air pour former un aérosol fortement chargé est étudié en laboratoire. On montre que, moyennant un certain nombre de dispositions, un déchargeur électrostatique capable d'évacuer d'un hélicoptère un courant de l'ordre de $60 \mu \mathrm{A}$ est réalisable. Les perturbations radioélectriques liées au fonctionnement de l'appareil devraient être négligeables.
\end{abstract}

\begin{abstract}
The problem of ejecting charges of one sign from an insulated body is considered. A system utilizing the condensation of the water vapor in air to form a charged aerosol has been studied in the laboratory. We have shown that, by using particular arrangements, it is possible to remove a current of $60 \mu \mathrm{A}$ from a helicopter. The radioelectric perturbations associated with the operation of the discharger should be negligible.
\end{abstract}

1. Introduction. - La réalisation d'hélicoptères tout temps, capables de prendre l'air quelles que soient les conditions météorologiques, est prévue pour le début des années 1980. Pour de tels appareils, les perturbations dues à l'accumulation d'électricité statique vont poser de sérieux problèmes. En effet, de même que l'avion, l'hélicoptère évoluant en atmosphère de nuage, de poussière ou de neige, se charge par le frottement des particules en suspension sur la surface de l'appareil.

L'accumulation de charges électriques d'une polarité dominante élève le potentiel $V_{\mathrm{H}}$ de l'hélicoptère isolé dans l'atmosphère. $\mathrm{Si}$ aucun processus limitatif n'intervenait, ce potentiel pourrait croître indéfiniment suivant la loi :

$$
\mathrm{d} V_{\mathrm{H}}=\frac{1}{C_{\mathrm{H}}} I_{\mathrm{c}} \mathrm{d} t
$$

où $C_{\mathrm{H}}$ est la capacité de l'hélicoptère et $I_{\mathrm{c}}$ l'intensité $\mathrm{du}$ courant tribo-électrique qui le charge.

En réalité, dès que $V_{\mathrm{H}}$ atteint une valeur critique $V_{\mathrm{Hc}}$ de quelques dizaines de kilovolts, des décharges en couronne s'allument spontanément sur les parties métalliques les plus saillantes de l'appareil. Il en résulte un courant de fuite vers l'atmosphère dont l'intensité $I_{\mathrm{f}}$ est sensiblement une fonction quadratique du potentiel $V_{\mathrm{H}}, I_{\mathrm{f}}=\operatorname{Cte}\left(V_{\mathrm{H}}-V_{\mathrm{Hc}}\right)^{2}$. En régime stationnaire ou lentement variable, celui-ci prendra donc une valeur d'équilibre qui correspond à l'égalité du courant de charge $I_{c}$ et du courant de fuite $I_{\mathrm{f}}$.
Du point de vue de l'utilisateur, deux inconvénients majeurs découlent de cette situation :

(i) Dans la plupart des cas, le courant d'effet couronne est pulsatoire (Impulsions de Trichel en polarité négative, streamers en polarité positive). Il en résulte un parasitage des instruments de radiocommunication et de radionavigation. Ceux-ci peuvent devenir inopérants lors de conditions météorologiques défavorables, alors que l'équipage en aurait le plus besoin. Qui plus est, la sensibilité de ces instruments à ce genre de perturbation est d'autant plus grande que l'on utilise des fréquences plus basses, ce qui est la tendance actuelle des dispositifs de radionavigation (radio-compas, systèmes OMEGA, LORAN, DECCA, etc...).

L'utilisation de déperditeurs passifs, pointes conductrices reliées à l'aéronef, est efficace sur les avions conventionnels du fait de l'entraînement des ions par l'air qui les balaye à une vitesse élevée $(\sim 200 \mathrm{~m} / \mathrm{s})$. Dans le cas de l'hélicoptère, les essais montrent que, même disposés aux extrémités des pales des rotors, les déperditeurs passifs classiques sont sans doute insuffisants lorsque les courants de charge sont très intenses (quelques centaines de microampères, voire quelques milliampères dans des conditions exceptionnelles). Ceci est dû au fait que le corps de la machine se déplace lentement et que les charges ne sont pas entraînées au loin.

(ii) Pour ces mêmes courants intenses et, en particulier, lorsque l'hélicoptère est en vol stationnaire, les potentiels d'équilibre $V_{\mathbf{H}}$ peuvent atteindre des 
valeurs de plusieurs centaines de kilovolts. Lorsque l'hélicoptère porte un fardeau suspendu par un filin métallique, la décharge de la capacité hélicoptère-sol peut occasionner des blessures par choc électrique au personnel de manutention au sol; il en est de même au cours d'opérations de sauvetage où l'utilisation d'un filin est nécessaire.

Pour des raisons de sécurité, l'emploi d'un fil conducteur traînant sur le sol pour assurer la mise à la terre de l'hélicoptère n'est pas toujours accepté. A la suite de manœuvres maladroites, une ligne pendante peut se prendre dans un rotor, avec les conséquences que l'on devine.

En vue de résoudre les problèmes électrostatiques sur les aéronefs à voilure tournante, certains auteurs ont proposé des systèmes à rétro-action (dits actifs) de contrôle du potentiel, ne faisant pas intervenir de liaisons matérielles entre l'hélicoptère et le sol [1,2].

Pour maintenir à une valeur suffisamment faible le potentiel d'un hélicoptère qui se charge continuellement, un système actif utilise dans son principe :

a) un détecteur répondant au champ électrique entourant l'appareil, généralement un voltmètregénérateur type moulin à champ ;

$b$ ) un générateur de charges unipolaires qui doit éjecter dans l'atmosphère un courant égal à celui qui charge l'appareil. Le signe et l'intensité du courant sont commandés par le signal électrique provenant du détecteur de champ.

La figure 1 montre schématiquement, dans le cas
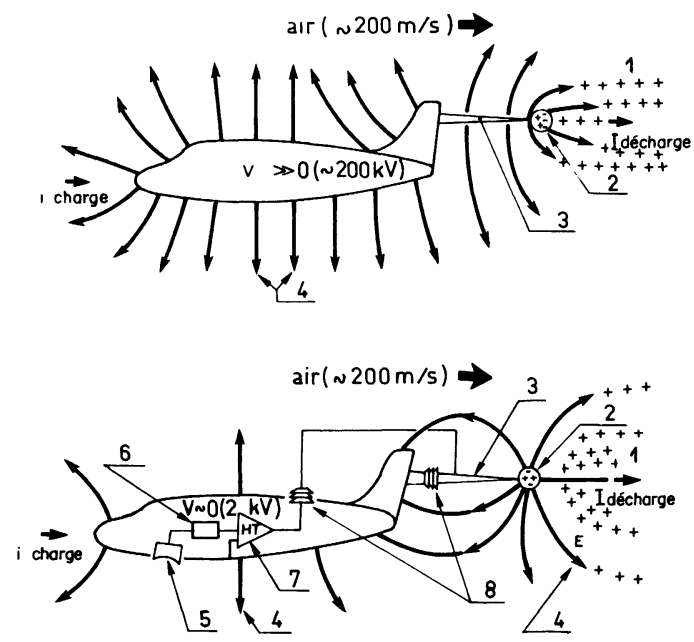

Fig. 1. - Principe des systèmes de décharges actif et passif. a) Système passif : $U \gg(\sim 200 \mathrm{kV}) ; b)$ Système actif : $U \sim 0(\mp 2 \mathrm{kV})$. 1. - Ions positifs entraînés par l'air à grande vitesse. 2. - Décharge en couronne-porteurs des deux signes. 3. - Pointe de décharge. 4. - Lignes de champ électrique. 5. - Mesureur de champ. 6. Amplificateur. 7. - Générateur haute tension $(\sim 200 \mathrm{kV})$. 8. - Isolateurs.

[Schematic of passive and active electrostatic discharge device. a) Passive device $U \gg(\sim 200 \mathrm{kV})$; b) Active device $U \sim 0(\mp 2 \mathrm{kV})$. 1. - Positive ions entrained by air at high speed. 2. - Corona discharge, carrying both signs. 3. - Discharge spike. 4. - Electric field lines. 5. - Field measuring device. 6. - Amplifier. 7. High voltage generator. 8. - Insulators.] d'un avion, la différence entre un procédé de champ passif (potentiel élevé de l'aéronef) et un procédé actif (potentiel très faible de l'aéronef).

Malheureusement, si l'idée paraît séduisante, les réalisations en ce domaine n'ont guère dépassé le stade expérimental ; en effet, les dispositifs employés pour l'éjection des charges unipolaires, à savoir, électrodes de décharge à très hautes tensions (centaines de kilovolts) (voir Fig. 1) ou éjecteurs de gouttelettes conductrices chargées par influence [3], sont lourds, complexes et, généralement, très insuffisants quant à l'intensité maximale du courant évacué (voir Chap. 5.2).

Afin de pallier ces difficultés, B. R. Whewell et al. [4], [5] proposent en 1970 d'appliquer à la décharge de l'électricité statique sur aéronef un procédé utilisé initialement par E. Baretto [6] pour les convertisseurs d'énergie de type électro gas dynamique. Il s'agit essentiellement de créer des particules chargées à très faible mobilité en condensant la vapeur d'eau contenue dans de l'air comprimé sur les ions gazeux issus d'une décharge en couronne.

Ce principe a été repris et analysé en détail à l'ONERA pour tenter de réaliser un déchargeur opérationnel, applicable à la stabilisation active du potentiel électrostatique des hélicoptères.

2. Le déchargeur à condensation d'air humide. 2.1 Principe. - Dans sa version initiale, le déchargeur est identique à celui présenté par B. R. Whewell et al. peut être décrit comme suit :

De l'air comprimé humide est détendu dans une petite tuyère supersonique (diamètre au col $2,3 \mathrm{~mm}$ ) débouchant à l'air libre.

La tuyère est métallique et au potentiel de la masse. Sur l'axe de la tuyère est disposée une aiguille portée à une haute tension positive ou négative suivant le signe des charges à éjecter. La pointe de l'aiguille, placée au col de la tuyère, est le siège d'une décharge en couronne sous l'effet de la tension de quelques kilovolts appliquée entre aiguille et tuyère. Des ions sont donc formés dans le flux gazeux et vont servir de noyaux de condensation pour la vapeur contenue dans l'air comprimé.

La baisse de température consécutive à la détente adiabatique de l'air humide dans une tuyère supersonique est telle que la vapeur est effectivement sursaturée au niveau de la décharge, à moins que l'air ne soit exceptionnellement sec.

La condensation sur les ions produit des microgouttelettes ou aérosols chargés. Leur diamètre est de l'ordre de quelques centaines d'angströms et leur charge de quelques charges élémentaires tout au plus, de telle sorte que leur mobilité est inférieure de deux ordres de grandeurs à celle des ions gazeux. Ceci signifie que, à la pression atmosphérique, la vitesse de dérive de ces gouttelettes par rapport à l'air, dans un champ électrique comparable au 
champ disruptif $\left(\sim 3 \times 10^{6} \mathrm{~V} / \mathrm{m}\right.$ à T.P.N. $)$, ne dépasse pas quelques mètres par seconde. La vitesse de l'air dans la tuyère étant supersonique, la dérive des gouttelettes est négligeable.

Nous mettons toutefois en évidence, au cours de l'étude, l'effet de la vitesse de dérive résiduelle lors du transport des particules par le jet en aval de la tuyère (cf. Chap. 4).

L'air comprimé, sous une pression de l'ordre de 6 bars (débit : $5 \mathrm{~g} / \mathrm{s}$ ) peut être fourni par la centrale pneumatique de l'hélicoptère. En outre, la tension nécessaire à l'entretien de la décharge en couronne ne dépasse pas $\pm 10 \mathrm{kV}$.

Le dispositif est donc tout à fait analogue à la partie éjecteur d'un convertisseur électrofluide dynamique, le collecteur étant situé à grande distance. En fait, la notion même de collecteur devient imprécise ; on pourrait la définir par la zone de l'espace à partir de laquelle une particule chargée ne retourne plus sur l'aéronef (cf. Chap. 4, § 3).

La figure 2 fournit un schéma de principe du déchargeur et indique la répartition des courants dans la pointe $\left(I_{\mathrm{p}}\right)$, dans la tuyère $\left(I_{\mathrm{T}}\right)$ et éjecté à l'infini $\left(I_{\mathrm{E}}\right)$.

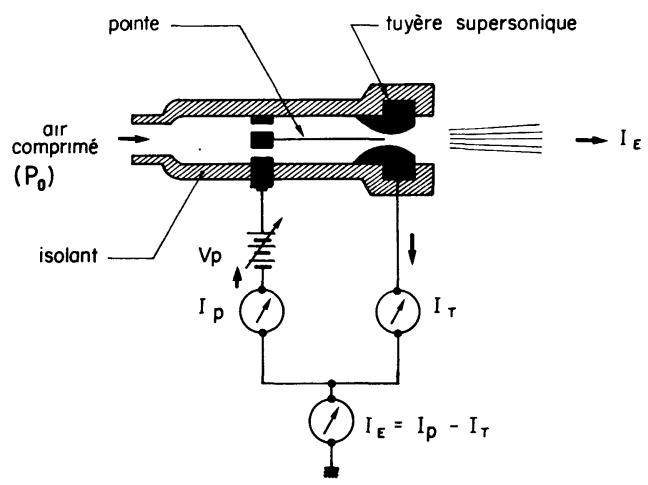

Fig. 2. - Déchargeur à condensation d'air humide.

[Wet air condensation discharger.]

2.2 DisPositiF EXPÉRIMENTAL. - Au cours de l'expérimentation, on s'efforce d'éloigner au maximum tous les conducteurs reliés au sol afin que les lignes de champ issues du jet ne soient pas trop différentes de ce que l'on obtiendrait sur l'aéronef en vol.

A cet effet, le déchargeur est disposé sur un mât isolant fixé au bord d'une table d'expérience, elle-même isolée du sol par des cales en verre.

La totalité de l'instrumentation électrique est disposée sur la table en amont du jet ; l'alimentation électrique se fait par des batteries $28 \mathrm{~V}$ de manière telle que la seule liaison électrique avec la masse $\mathrm{du}$ laboratoire passe par l'intermédiaire du microampèremètre servant à la mesure du courant éjecté $I_{\mathrm{E}}$ (Fig. 3).

Un éventuel courant qui reviendrait du déchargeur vers la table d'expérience n'est donc pas lu sur le

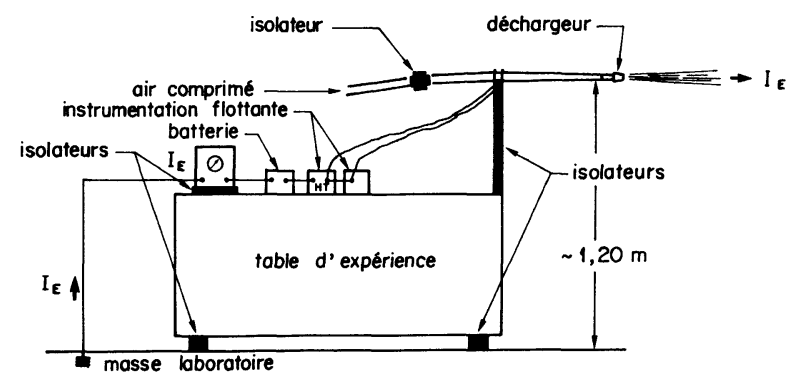

Fig. 3. - Implantation du dispositif expérimental.

[Experimental set up.]

micro-ampèremètre (on peut vérifier que ce courant est négligeable en reliant directement la table à la masse électrique du laboratoire). $\mathrm{Du}$ point de vue électrique, la table d'expérience simule donc l'aéronef à décharger.

Il est important de travailler dans l'obscurité, afin de détecter les décharges susceptibles de neutraliser le jet (cf. Chap. 4).

2.3 EXPÉRIENCES PRÉLIMINAIRES. - La pointe émettrice, localisée au col de la tuyère, est polarisée positivement. Pour une pression absolue de 6 bars à l'entrée du déchargeur, le courant $I_{\mathrm{p}}$ maximal qui circule dans la pointe, juste avant l'apparition du claquage (étincelle) électrique entre pointe et tuyère, vaut environ $70 \mu \mathrm{A}$. Ce courant se répartit de la manière suivante :

- courant pointe $: I_{\mathrm{p}} \sim 70 \mu \mathrm{A}$,

- courant tuyère : $I_{\mathrm{T}} \sim 65 \mu \mathrm{A}$,

- courant éjecté : $I_{\mathrm{E}} \sim 5 \mu \mathrm{A}$.

Par ailleurs, on constate qu'une grille ou une pointe métallique (collecteurs), située dans le jet à quelques centimètres de la tuyère et reliée à la masse du laboratoire, recueille la quasi-totalité du courant émis par la pointe ; le courant $I_{\mathrm{T}}$ revenant sur la tuyère étant alors voisin de zéro, on obtient, avec collecteur :

- courant pointe $: I_{\mathrm{p}} \sim 70 \mu \mathrm{A}$,

- courant tuyère $: I_{\mathrm{T}} \sim 0$,

- courant collecteur : $I_{\mathrm{C}} \sim 70 \mu \mathrm{A}$.

En polarité négative, le comportement est comparable, mais le courant $I_{\mathrm{p}}$ maximal (avant claquage pointe tuyère) est beaucoup plus intense $(\sim 300 \mu \mathrm{A})$ et le courant sur la tuyère ne s'annule pas en présence $\mathrm{du}$ collecteur. Celui-ci reçoit un courant maximal de l'ordre de $100 \mu \mathrm{A}$.

Dans un but de contrôle, les essais furent réalisés indépendamment à l'ONERA et au Laboratoire d'Electrostatique du CNRS de Grenoble. Les résultats sont très similaires [7].

Dans ces conditions, compte tenu de l'intérêt de ce type d'appareil, une étude approfondie du déchargeur a été entreprise pour tenter de déterminer pourquoi et comment en l'absence de collecteur 
rapproché, la grande majorité des charges sortant de la tuyère retourne sur celle-ci ou sur des objets conducteurs voisins.

Par ailleurs, en raison des lois de l'électrostatique et de l'aérodynamique, il paraît certain que le fonctionnement interne de la tuyère ne dépend pas de ce qui se passe à l'extérieur. C'est ce fonctionnement interne que nous allons tout d'abord étudier.

3. L'éjecteur, étude expérimentale. - L'éjecteur - zone de création des charges - est défini comme l'ensemble pointe-tuyère.

Lors de l'étude spécifique de l'éjecteur, un collecteur métallique est disposé dans le jet, à un centimètre environ du plan de sortie de la tuyère (Fig. 4). Le collecteur se compose d'une électrode cylindrique creuse sur l'axe de laquelle est disposée une aiguille pointue. On constate que la décharge en couronne qui apparaît sur la pointe de cette aiguille sous l'effet du champ de charge de l'espace, neutralise totalement les charges unipolaires transportées par le jet. Cette neutralisation est en elle-même une indication de l'importance de la charge d'espace au sein du jet. E. Baretto et al. ont montré que cet effet n'apparaît qu'à la condition que les porteurs soient effectivement des gouttelettes à faible mobilité [6].

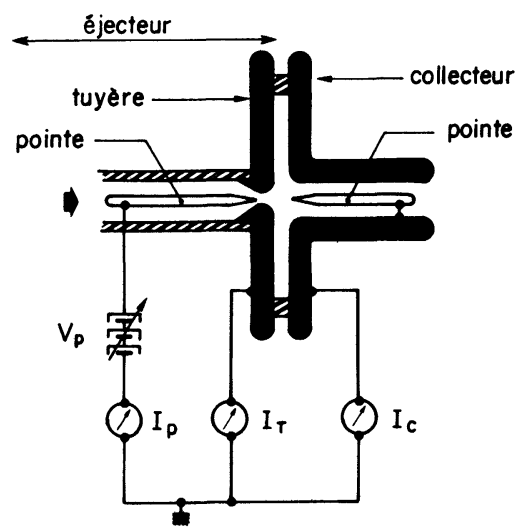

Fig. 4. - Schéma de l'expérience. Etude de l'éjecteur.

[Ejector study. Schematic of the experiment.]

On s'intéresse au courant $I_{0}$ maximal qui peut franchir le plan de sortie de la tuyère. Ce courant $I_{0}$ est identique au courant $I_{\mathrm{c}}$ reçu par le collecteur, pourvu que celui-ci soit au potentiel zéro et situé suffisamment près de l'embouchure de la tuyère, dans l'axe du jet (distance éjecteur-collecteur inférieure à $1 \mathrm{~cm})$, figure 4 .

Une limite supérieure du courant est obtenue en écrivant que sur un cylindre de rayon $R$, le champ $E_{\mathrm{R}}$ dû à la charge d'espace et qui vaut : $E_{\mathrm{R}}=I_{0} / 2 \pi \varepsilon_{0} v R$ ( $v$ étant la vitesse de l'écoulement identique pour le fluide moteur et les charges électriques) atteint la valeur disruptive $E_{\mathrm{D}}$,

$$
I_{0 \max } \leqslant 2 \pi E_{\mathrm{D}} v \varepsilon_{0} R \text {. }
$$

La valeur réelle de $I_{0 \max }$ se situe, dans ce type de générateur, autour de $30 \%$ de la borne supérieure. D'une part, la pression locale - et donc la valeur du champ disruptif - décroît vers l'aval au cours de la détente supersonique (effet en partie compensé par l'accroissement de la vitesse et du rayon). D'autre part, le champ $E_{\mathrm{R}}$ est en réalité la somme du champ de charge d'espace et du champ de polarisation nécessaire à l'entretien de la décharge en couronne sur la pointe.

Bien que $I_{0 \max }$ soit inférieur à la valeur calculée, la formule ci-dessus donne les lois de similitude exactes et aisément vérifiables :

1) pour deux écoulements géométriquement semblables, le courant maximal varie proportionnellement au rayon de la tuyère (et non à sa section) ;

2) le courant maximal mesuré est, pour une géométrie donnée, proportionnel à la pression motrice (par l'intermédiaire de $E_{\mathrm{D}} \propto P$, car $v$ est indépendant de la pression motrice pour un écoulement supersonique).

3.1 Polarité Positive. - En polarité positive, et en restant sur le plan qualitatif, le fonctionnement de l'éjecteur se conçoit en considérant la répartition des courants - juste avant l'étincelle - en fonction de la position axiale de la pointe dans la tuyère, ainsi que l'évolution de la tension de polarisation $V_{\mathrm{p}}$ maximale correspondante (Fig. 5). L'origine des abscisses est prise au col de la tuyère. En éloignant la pointe vers l'amont du col, la tension maximale $V_{\mathrm{p}}$ croît (augmentation de la pression locale et de la distance pointe-tuyère). Le courant $I_{\mathrm{p} \max }$ augmente légèrement, mais l'on assiste à une décroissance du courant collecteur $I_{\text {c } \max }$, liée à l'apparition d'un courant de retour $I_{\mathrm{T}}$ sur la tuyère. Il est évident que si l'on éloigne progressivement la décharge en couronne de la zone de sursaturation (laquelle débute pratiquement au niveau du col), une fraction

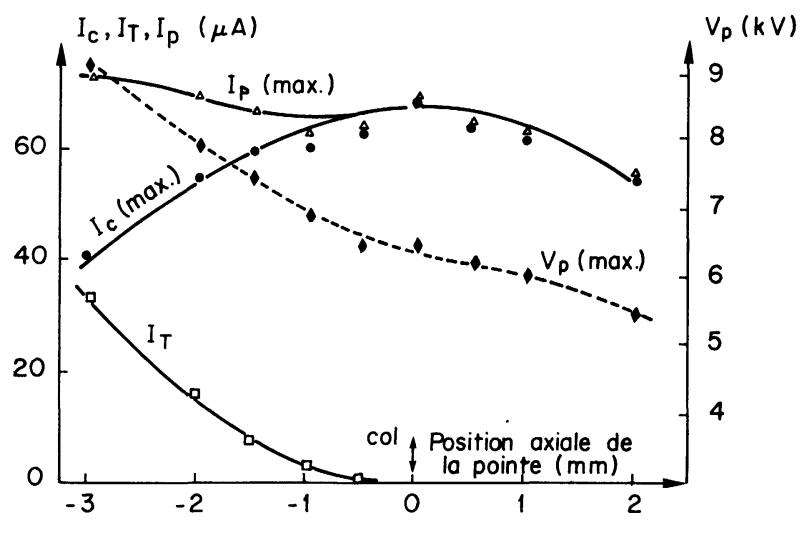

tuyère convergente-divergente; $\phi$ col $=2,3 \mathrm{~mm} ; P_{0}=6$ bars

Fig. 5. - Fonctionnement de l'éjecteur. Polarité positive. Courants maximum en fonction de la position axiale de l'aiguille.

[Ejector study. Positive polarity. Maximum currents versus axial position of the needle.] 
croissante des ions gazeux émis par la décharge va être captée par la tuyère avant de pénétrer dans cette zone.

$\mathrm{Si}$, par contre, on avance vers l'aval la pointe dans le divergent de la tuyère, les courants $I_{\mathrm{p} \text { max }}$ et $I_{\mathrm{c} \max }$ restent égaux $\left(I_{\mathrm{T}}=0\right)$. La décroissance de la tension et des courants maximaux est alors liée à la chute de pression statique qui se produit au cours de la détente supersonique, le long du divergent.

L'aspect visuel de la décharge positive est celui d'un minuscule point brillant à l'extrémité de la pointe, il s'agit donc du régime décrit par Hermstein [8], comme le démontre par ailleurs l'absence d'impulsions dans le courant de la pointe. Lorsqu'un streamer se produit, il dégénère en étincelle.

3.2 Polarité NÉgative. - Considérons les courbes portées sur la figure 6 représentant les courants $I_{\mathrm{p}}$, $I_{\mathrm{T}}, I_{\mathrm{c}}$ en fonction de la polarisation négative $V_{\mathrm{p}}$ de la pointe. La pression motrice absolue est de 6 bars et la pointe est située au col de la tuyère. Jusqu'à une tension de $6 \mathrm{kV}$, les courants $I_{\mathrm{p}}$ et $I_{\mathrm{c}}$ sont égaux $\left(I_{\mathrm{T}}=0\right)$ et croissent linéairement jusqu'à environ $80 \mu \mathrm{A}$, avec la tension appliquée $V_{\mathrm{p}}$. Au-delà de $6 \mathrm{kV}$, un courant $I_{\mathrm{T}}$ apparaît et augmente très rapidement avec $V_{\mathrm{p}}$. Simultanément, la croissance de $I_{\mathrm{c}}$ se ralentit. Celui-ci atteint la valeur maximale de $100 \mu \mathrm{A}$ puis décroît jusqu'à l'apparition d'étincelles pour $V_{\mathrm{p}} \sim 10 \mathrm{kV}$.

L'aspect de la décharge négative est celui d'une zone lumineuse entourant la pointe et dont l'extension et l'éclat croissent avec $V_{\mathrm{p}}$. Lorsque $V_{\mathrm{p}}$ atteint

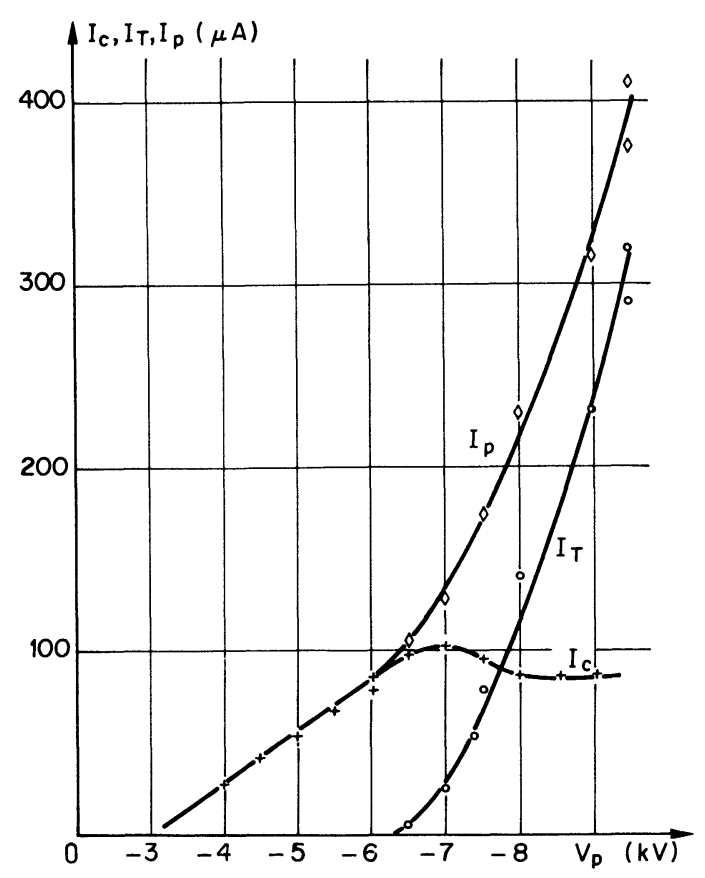

Fig. 6. - Fonctionnement de léjecteur. Polarité négative. Courants $I_{\mathrm{p}}, I_{\mathrm{c}}, I_{\mathrm{T}}$ en fonction de la tension pointe.

[Ejector study. Negative polarity. Currents $I_{\mathrm{p}}, I_{\mathrm{c}}, I_{\mathrm{T}}$ versus needle potential.]
$6 \mathrm{kV}$, la luminosité occupe une portion notable de l'intervalle pointe-tuyère.

La décroissance observée du courant $I_{\mathrm{c}}$ et la croissance de $I_{\mathrm{p}}$ s'expliquent par le fait que la décharge négative produit des électrons qui ne peuvent servir de site de nucléation. C'est seulement si ces électrons ont le temps de se convertir en ions négatifs $\mathrm{O}_{2}^{-}$ par attachement que la nucléation peut avoir lieu. Lorsque le champ est élevé, il est vraisemblable que la majorité des électrons atteint la paroi de la tuyère avant attachement.

Le fait qu'en l'absence de collecteur proche, les charges électriques retournent sur la tuyère en suivant des trajectoires situées en aval de son embouchure, peut être illustré par l'expérience décrite par la figure 7 : une électrode métallique $\mathrm{D}$ munie d'une ouverture pour l'écoulement du jet et disposée juste après l'éjecteur en masquant celui-ci, reçoit la quasitotalité du courant de retour, c'est-à-dire $I_{\mathrm{D}} \simeq I_{\mathrm{p}}-I_{\mathrm{E}}$; $I_{\mathrm{T}} \simeq 0$.

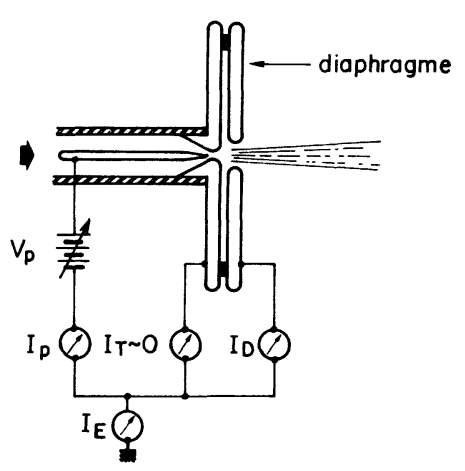

Fig. 7. - Détection du courant de recirculation. Dispositif expérimental.

[Return current detection. Experimental set up.]

4. Transport des charges par le jet libre. - 4.1 CONSIDÉRATIONS AÉRODYNAMIQUES. - A la sortie de la tuyère, la détente de l'air se poursuit en ce qu'il est convenu d'appeler un jet sous-détendu.

On peut $y$ distinguer trois zones principales (Fig. 8) :

a) un noyau central supersonique comportant un grand nombre d'ondes de choc stationnaires et qui disparaît après une longueur axiale égale à quelques dizaines de fois le diamètre de sortie de la tuyère ;

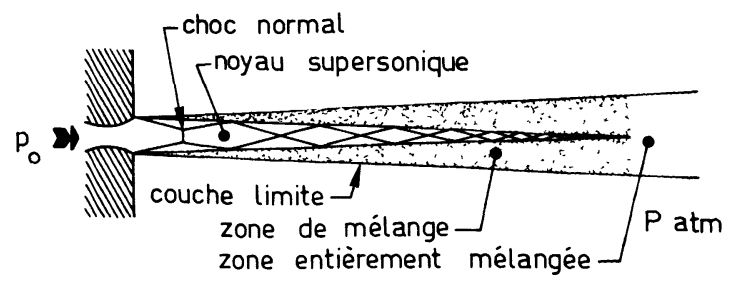

Fig. 8. - Jet supersonique.

[Supersonic jet.] 
b) une zone subsonique turbulente entourant ce noyau et dans laquelle l'air issu de la tuyère se mélange à l'air ambiant ;

c) le noyau est prolongé en aval par une zone subsonique turbulente contenant à la fois l'air de la tuyère et l'air ambiant entraîné.

4. 2 NeUtralisation DU JET PAR LES DÉCHARgeS EN COURONNE SUR LA TUYÈRE. - En faisant fonctionner le premier prototype en jet libre dans l'obscurité totale, on a observé la présence de nombreuses décharges en couronne sur les parties externes de l'éjecteur.

Ces décharges en couronne sont provoquées par le champ dû à la charge du jet. Elles vont neutraliser plus ou moins complètement le jet, ce qui réduit beaucoup l'efficacité globale du déchargeur.

Remarquons enfin que le courant transporté par ces décharges est équivalent à un courant de retour ; la seule indication permettant de distinguer un courant de charges négatives dû à une décharge en couronne sur la tuyère, d'un courant de charges positives issues $\mathrm{du}$ jet et retournant sur l'éjecteur, est fournie par l'aspect lumineux de la tuyère en fonctionnement.

Afin d'éliminer ces décharges parasites, la forme extérieure de la tuyère a été reprise. Sans modifier le conduit d'écoulement, son diamètre extérieur est porté à $120 \mathrm{~mm}$. Les bords sont arrondis et les fils d'alimentation débouchent sur la face arrière.

On constate alors la disparition de tout point lumineux lorsque le déchargeur fonctionne en jet libre ; toutefois, le courant éjecté $I_{\mathrm{E}}$ n'augmente pas dans des proportions considérables :

de $I_{\mathrm{E}}=3$ à $5 \mu \mathrm{A}$, on passe à $I_{\mathrm{E}}=6$ à $10 \mu \mathrm{A}$,

ce qui tend à prouver que la neutralisation par les décharges en couronne sur la tuyère n'est pas seule en cause dans les processus limitant l'efficacité du déchargeur.

4.3 RETOUR DES CHARGES PAR FUITES RADIALES AUTOUR DU JET. - 4.3.1 Modélisation du processus de retour. - On considère le modèle simplifié où le jet est assimilé à un écoulement uniforme, semi-infini, de rayon $r$ et de vitesse constante $v$ (Fig. 9) ; $x$ est la distance séparant un point considéré du jet du plan de sortie de la tuyère et $\rho(x)$ est la densité de charge

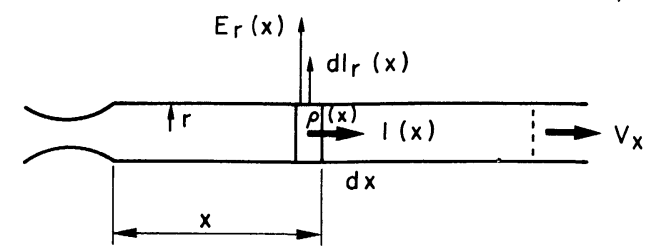

Fig. 9. - Modélisation des fuites radiales.

[Radial losses modelisation.] supposée fonction de $x$ seulement. Le champ radial à la périphérie du jet est :

$$
E_{\mathrm{r}}(x)=\frac{\rho(x) r}{2 \varepsilon_{0}}
$$

en négligeant la vitesse de dérive axiale des particules chargées, le courant transporté par le jet devient :

$$
I(x)=\rho(x) .2 \pi r . v .
$$

On évalue le courant $I_{\mathrm{r}}$ qui s'échappe radialement du jet en considérant le mouvement des particules chargées qui sont au voisinage de sa périphérie pour une tranche $\mathrm{d} x$ du jet.

Si $\mu$ est leur mobilité :

$$
\mathrm{d} I_{\mathrm{r}}(x)=\mu(I(x))^{2} \mathrm{~d} x / \varepsilon_{0} \pi r^{2} \cdot v^{2} .
$$

En intégrant l'équation différentielle (3), on obtient la valeur du courant restant dans le jet au point d'abscisse $x$ :

$$
I(x)=I(0) /\left(1+\frac{\mu I(0) x}{\varepsilon_{0} \pi r^{2} v^{2}}\right) .
$$

En remplaçant $I(x)$ par $\rho(x)$, on obtient la relation :

$$
\rho(x)=\rho(0) /\left(1+\frac{\mu \rho(0) x}{\varepsilon_{0} v}\right)
$$

qui montre que l'évolution de la densité de charges est indépendante des dimensions géométriques de l'écoulement [9].

Les charges qui sortent radialement du jet suivent alors les lignes de force du champ électrique. La mesure $\mathrm{du}$ potentiel autour du jet [10] montre que ces dernières se répartissent dans l'espace comme l'indiquent les figures 10 et 13 . En amont d'un point $X_{0}$, les lignes de champ issues du jet retournent sur l'éjecteur. En aval de ce point, les lignes vont à l'infini.

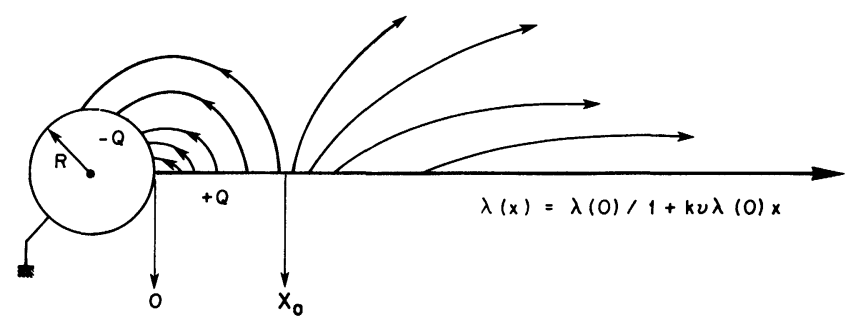

Fig. 10. - Représentation schématique des lignes du champ électrique. Configuration : jet libre-tuyère au potentiel zéro.

[Schematic of electric field lines. Free jet, nozzle at zero potential.]

L'abscisse du point $X_{0}$ de non-retour des particules chargées peut être déterminée si l'on représente l'éjecteur par une sphère conductrice au potentiel de la masse et de rayon $R>r$. Le jet est assimilé à une ligne chargée dont la densité linéique $\lambda(x)$ se déduit de (4) par la relation :

$$
I(x)=\lambda(x) \cdot v .
$$


On peut alors calculer la charge image $-Q$ portée par la sphère sous l'influence électrostatique du jet, soit :

$$
\begin{aligned}
-Q & =-\int_{0}^{\infty} \frac{R}{R+x} \lambda(x) \mathrm{d} x \\
& =-\frac{\lambda(0) R}{1-k \lambda(0) v \cdot R} \cdot \ln k \lambda(0) v R
\end{aligned}
$$

avec $K=\mu / \pi \varepsilon_{0} r^{2} v^{2}$.

Or, les tubes de champ qui se referment sur la sphère ne peuvent provenir que d'une portion du jet, comprise entre les abscisses 0 et $X_{0}$, dont la charge totale est $+Q$. Le point de non-retour $X_{0}$ répond donc à la relation :

$$
\int_{0}^{x_{0}} \lambda(x) \mathrm{d} x=Q
$$

soit d'après (7) :

$$
X_{0}=\frac{1}{k \lambda(0) v}\left[(k \lambda(0) v R)^{\frac{k \lambda(0) v R}{\lambda \lambda(0) v R-1}}-1\right] .
$$

Le courant éjecté $I_{\mathrm{E}}$, en fonction du courant à l'origine $I(0)=I_{\mathrm{p}}$ s'écrit d'après (4) et (9) :

$$
\begin{aligned}
I_{\mathrm{E}} & =I\left(X_{0}\right)=\frac{I(0)}{1+k I(0) X_{0}} \\
& =I(0)\left[(k I(0) R)^{\frac{k I(0) R}{1-k I(0) R}}\right] .
\end{aligned}
$$

La comparaison entre le relevé expérimental de $I_{\mathrm{E}}=f\left(I_{\mathrm{p}}\right)$ et la courbe théorique normalisée issue de la formule (10) est présentée sur la figure 11. La bonne concordance observée pourrait toutefois être fortuite, compte tenu des nombreuses hypothèses simplificatrices retenues pour l'établissement du modèle théorique.

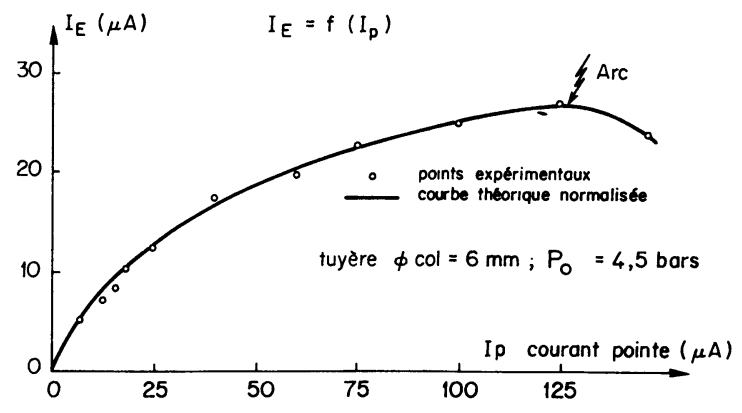

Fig. 11. - Comparaison expérience-théorie.

[Experimental and theorical results.]

Plus significatives, sans doute, sont les prévisions qui découlent des expressions (9) et (10), quant à l'influence du rayon extérieur $R$ de l'éjecteur sur l'allure des courbes $I_{\mathrm{E}}=f\left(I_{\mathrm{p}}\right)$.
4.3.2 Influence des dimensions caractéristiques externes de l'éjecteur. - D'après la relation (3), pour une valeur donnée du courant $I(0)=I_{\mathrm{p}}$ à l'origine, l'abscisse du collecteur fictif défini par $X_{0}$ est toujours fonction croissante du rayon $R$ de l'éjecteur. Autrement dit, la longueur du jet pour laquelle les lignes de champ se referment sur la tuyère décroît et tend vers zéro lorsque l'on réduit les dimensions géométriques de l'éjecteur.

Dans ces conditions, si l'on considère un éjecteur de plus en plus petit, on s'attend à une augmentation de la pente des courbes $I_{\mathrm{E}}=f\left(I_{\mathrm{p}}\right)$; celles-ci devant atteindre la droite limite $I_{\mathrm{E}}=I_{\mathrm{p}}$ pour la valeur théorique $R=0$ (d'après la formule (10)).

D'un autre côté, le champ électrique moyen à la surface de la tuyère, que l'on peut définir comme

$$
\bar{E}=\frac{Q}{\varepsilon_{0}} \frac{1}{4 \pi R^{2}},
$$

s'écrit d'après (7) :

$$
\bar{E}=\frac{1}{4 \pi \varepsilon_{0} R^{2}}\left[\frac{\lambda(0) R}{1-k \lambda(0) v R} \ln k \lambda(0) R\right] .
$$

La fonction $\bar{E}$ est par contre toujours décroissante avec $R$. Le champ à la surface augmente si le rayon de la tuyère diminue (ceci signifie globalement que l'effet de concentration du champ par l'électrode métallique l'emporte sur la diminution de l'influence électrostatique tuyère-jet avec la diminution de $R$ ). Il est donc prévisible que le seuil d'apparition des décharges en couronne neutralisantes à la surface de l'éjecteur sera atteint par un courant $I(0)$ d'autant plus faible que $R$ est réduit.

Les relevés expérimentaux de $I_{\mathrm{E}}=f\left(I_{\mathrm{p}}\right)$ pour $R$ variant de 10 à $60 \mathrm{~mm}$ sont portés sur la figure 12 . Les courbes illustrent parfaitement les phénomènes décrits ci-dessus. Les maxima des courbes correspondent à l'apparition des décharges en couronne

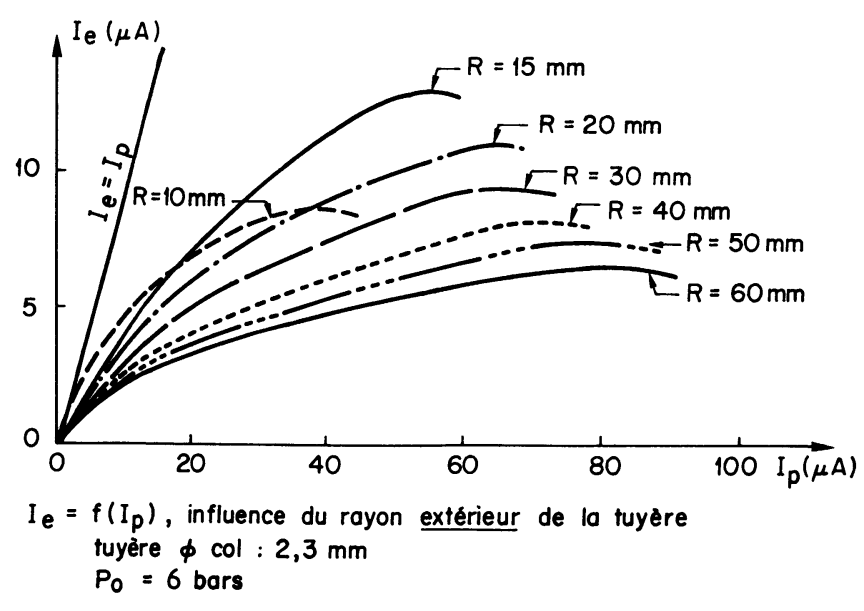

Fig. 12. - Déperditeur en jet libre. Influence des dimensions externes de la tuyère.

[Free jet discharger. Role of external dimensions of the nozzle.] 
à la surface de la tuyère. L'optimum se situe pour un rayon extérieur $R \simeq 15 \mathrm{~mm}$. Celui-ci est toutefois peu marqué et le courant maximal éjecté $I_{\mathrm{E}}$ ne dépasse pas $15 \mu \mathrm{A}$.

5. Tentatives d'amélioration des performances du déchargeur en jet libre. - 5.1 INFLUENCE D'UN ÉCOULEMENT D'AIR DE FAIBLE VITESSE. - La connaissance du fonctionnement de l'appareil, telle que nous l'avons exposée au cours des chapitres précédents et qui est résumée par la figure 13 fait entrevoir

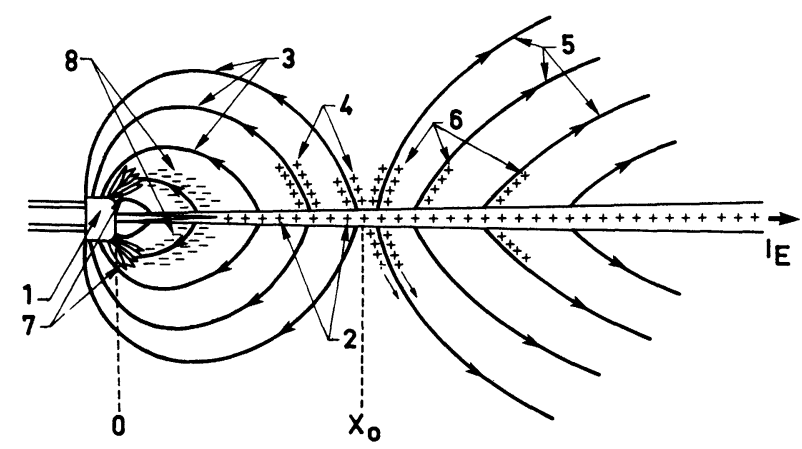

Fig. 13. - Déperditeur en jet libre : représentation schématique du fonctionnement. 1. - Ejecteur au potentiel zéro. 2. - Jet chargé positivement. 3. - Lignes de champ issues du jet et retournant sur l'éjecteur. 4. - Gouttelettes chargées diffusant hors du jet, s'évaporant et retournant sur l'éjecteur sous forme d'ions gazeux. 5. - Lignes de champ issues du jet et se dirigeant vers l'infini. 6. - Gouttelettes chargées issues du jet et se dirigeant vers l'infini en s'évaporant. 7. - Décharges en couronne à la surface externe de l'éjecteur métallique. 8. - Charges négatives produites par des décharges en couronne (7) et neutralisant partiellement le jet chargé (2).

[Free jet discharger. Schematic of its functionning. 1. - Ejector at potential zero. 2. - Positively charged jet. 3. - Field lines issued from jet and returning to ejector. 4. - Charged droplets, scattering outside the jet, evaporating and returning to ejector in the from of gazeous ions. 5. - Field lines issued from jet and going to infinity. 6. - Charged droplets issued from jet and going to infinity while evaporating. 7. - Corona discharge at the external surface of metallic ejector. 8. - Negative charges produced by Corona discharged (7) and partially neutralizing the charged jet (2).]

un certain nombre de possibilités visant à améliorer l'efficacité de l'éjection des charges unipolaires.

En premier lieu, on peut penser que le fait de disposer les déchargeurs dans un écoulement d'air de forte section, dont la vitesse est parallèle au jet, devrait permettre d'entraîner toutes les charges qui reviennent du jet sur la tuyère. Il suffirait alors théoriquement d'utiliser un éjecteur de dimensions externes suffisantes (de manière à éviter les décharges induites), pour obtenir un rendement $I_{\mathrm{E}} / I_{\mathrm{p}}$ voisin de l'unité. L'écoulement en question existe en fait sur l'hélicoptère sous forme du flux aérodynamique descendant du rotor principal.

Les essais réalisés en soufflerie montrent effectivement une influence positive d'un écoulement secondaire sur l'efficacité du déchargeur. Celle-ci est toutefois moins marquée que l'on aurait pu s'y attendre, compte tenu de la faible mobilité présumée des particules chargées. Une vitesse de l'écoulement externe auxiliaire de l'ordre de $40 \mathrm{~m} / \mathrm{s}$ est nécessaire pour obtenir le doublement du courant éjecté $I_{\mathrm{E} \max }$. Il est très vraisemblable que les gouttelettes qui diffusent radialement hors du jet s'évaporent rapidement et reviennent sur l'éjecteur sous forme d'ions gazeux à mobilité élevée. Dans ces conditions, il serait intéressant de changer la nature des porteurs de charges et d'utiliser, par exemple, des gouttelettes d'eau plus volumineuses qui devraient mettre un temps plus important pour s'évaporer.

5.2 UTILISATION D'UN AÉROSOL DE GOUTTELETTES CHARGÉES PAR INFLUENCE. - La pointe à décharge du déperditeur est remplacée par une aiguille creuse reliée en amont à un réservoir pressurisé contenant de l'eau salée. Le tout est porté à la haute tension réglable $V$. La pointe de l'aiguille est disposée au col de la tuyère. Lorsque celle-ci n'est pas alimentée en eau, une décharge en couronne apparaît à la pointe de l'aiguille. Le déchargeur est alors identique à celui décrit aux chapitres précédents. Lorsque le réservoir d'alimentation est porté à une pression suffisante, un aérosol fortement chargé se forme à l'extrémité de l'aiguille.

On peut alors comparer les deux modes de création de charges.

La tuyère utilisée, de dimensions extérieures suffisantes, est telle que les décharges induites à sa surface ne se manifestent pas lorsque les porteurs de charges sont créés par condensation (cf. Chap. 4.3).

Lorsque le débit d'eau est suffisant, on constate que le courant $I_{\mathrm{p}}$ est pratiquement égal au courant éjecté $I_{\mathrm{E}}$ jusqu'à une valeur de l'ordre de $14 \mu \mathrm{A}$. Après quoi, l'augmentation de la tension $V_{\mathrm{p}}$ est sans influence sur l'intensité du courant éjecté $I_{\mathrm{E}}$.

L'explication de ce comportement est la suivante :

Lors de la mise sous tension de l'aiguille, une pellicule d'eau apparaît immédiatement sur la surface externe de la tuyère. Sous l'effet du champ électrique, cette pellicule forme des petites pointes (cônes de Taylor) au niveau des lèvres de la tuyère. L'expérimentateur qui prend soin de travailler dans l'obscurité complète peut alors constater que ces pointes liquides s'illuminent dès que le courant éjecté atteint la valeur critique $I_{\mathrm{E}} \simeq 14 \mu \mathrm{A}$. Les décharges en couronne sur les cônes de Taylor s'opposent donc à toute augmentation supplémentaire du courant transporté par le jet. Il est à noter que cette valeur critique est celle obtenue par Buser et al. qui étudièrent un déchargeur pour hélicoptère fondé sur ces principes [3].

5.3 INFLUENCE DE LA FORME EXTÉRIEURE DE L 'ÉJECTEUR. - Par contre, en revenant à un dispositif à condensation d'air humide, la question se pose de savoir si l'on peut optimiser la forme extérieure de la tuyère, de manière à retarder l'apparition des décharges en couronne induites, tout en gardant 
une dimension caractéristique $R$ réduite de l'éjecteur (faible recirculation des charges).

Une optimisation à ce niveau a donc été tentée, mais elle est peu efficace, comme l'illustrent les photographies de la figure 14, à savoir :

Figure $14 a$ : la tuyère, de $1 \mathrm{~cm}$ de rayon extérieur, est de forme hémisphérique; pour un courant $I_{\mathrm{p}} \sim 70 \mu \mathrm{A}$, la décharge corona apparaît au niveau de la sortie de la tuyère, au sommet de l'hémisphère.

Figure $14 b$ : pour réduire la concentration du champ à la sortie de la tuyère, celle-ci a maintenant une forme cylindrique et la décharge se situe sur le bord arrondi du cylindre.

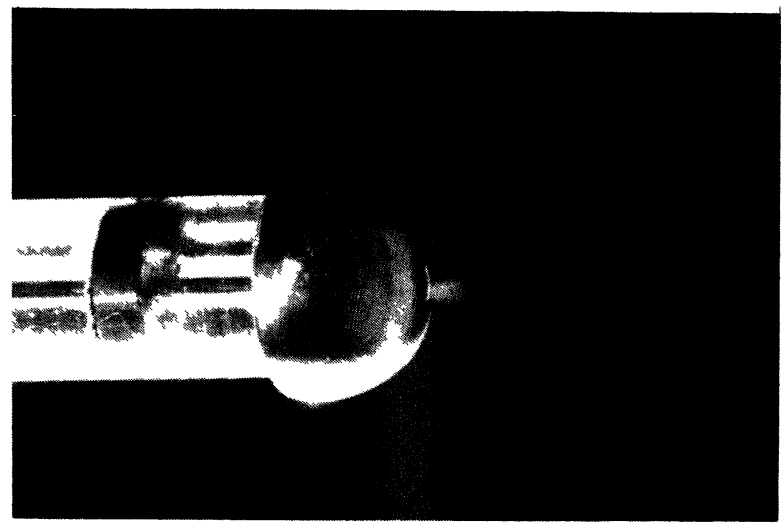

a)

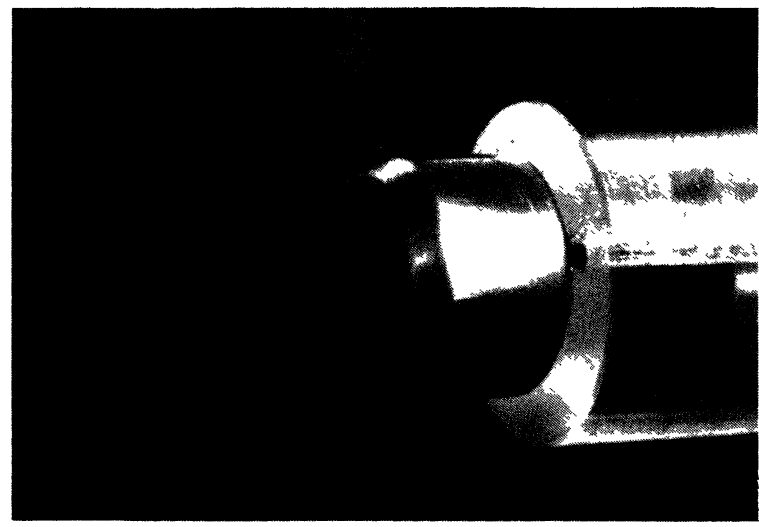

b)

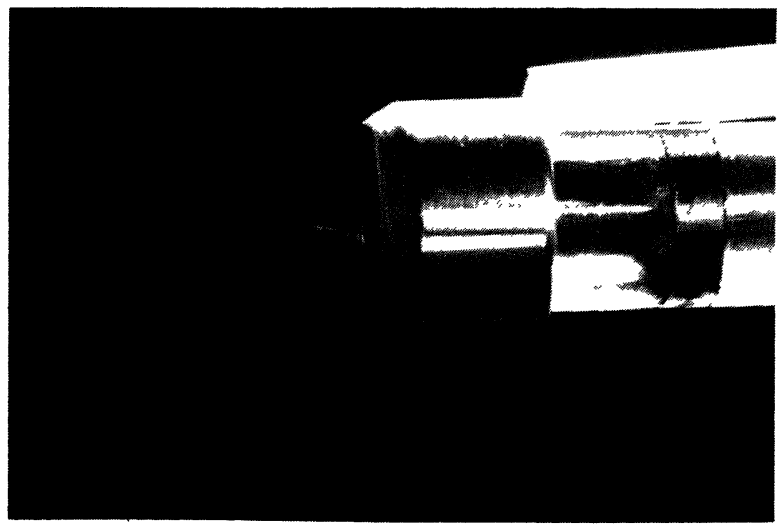

c)

Fig. 14. - Décharges induites sur l’éjecteur

[Induced discharges on the ejector.]
Figure 14c : ayant soupçonné le manchon de plexiglass, qui protège les fils de liaisons, de se charger et d'augmenter le champ électrique sur le bord du cylindre où apparaissent les décharges, celui-ci a été retiré. On constate alors l'illumination du jet lui-même, laquelle fait apparaître la structure du noyau.

Il est probable que cette décharge est favorisée par le très fort gradient de concentration des charges qui se manifeste au niveau du choc normal [11]. A ce niveau, il semble impossible d'optimiser davantage la forme de l'éjecteur.

Dans ces trois configurations, le courant éjecté $I_{\mathrm{E}}$ est du même ordre et vaut sensiblement $10 \mu \mathrm{A}$.

En résumé, on peut dire que ni l'optimisation de la forme et des dimensions caractéristiques de l'éjecteur, ni l'utilisation d'un double flux ou de porteurs de charges de dimensions accrues n'ont mis en évidence un gain véritablement substantiel de l'efficacité de décharge de l'appareil. Seule, apparemment, la méthode décrite au paragraphe suivant présente une action spectaculaire à ce point de vue.

5.4 TRANSPORT DES CHARGES ÉLECTRIQUES PAR UN ÉCOULEMENT PARTIELLEMENT GUIDÉ. - Pour éviter à la fois les décharges induites sur la tuyère et la recirculation des charges par l'extérieur du jet, le déchargeur, toujours disposé à l'extrémité de la canne isolante, est entièrement recouvert par un carénage isolant. Seul un canal cylindrique de $3 \mathrm{~mm}$ de diamètre et $30 \mathrm{~mm}$ de longueur, percé dans l'isolant (plexiglass) assure la communication entre la sortie de la tuyère et l'extérieur. La longueur $l$ du canal est déterminée empiriquement. Il semble que, dans cette configuration également, la présence d'un optimum pour la longueur $l$ du canal, traduit le conflit des deux effets :

- si le canal est trop long, les fuites radiales à l'intérieur du canal prennent une importance prépondérante,

- si le canal est trop court, les charges qui stagnent à la surface externe de l'isolant et dans l'air en aval de l'orifice de sortie du canal sont trop proches de la tuyère métallique, d'où un champ électrique axial trop intense.

Ces deux phénomènes se traduisent par un même effet, à savoir l'apparition de décharges rampantes à l'intérieur du canal. Comme le montre la photographie de la figure 15, ces décharges neutralisent non seulement les charges électriques déposées sur la paroi interne du canal, mais se prolongent dans l'air sous forme de streamers pour neutraliser la charge d'espace en aval du déchargeur. Lorsque le carénage est en place, le courant maximal éjecté se situe néanmoins entre 35 et $45 \mu \mathrm{A}$, ce qui constitue un gain très appréciable par rapport au déchargeur en jet libre $\left(I_{\mathrm{E} \max } \simeq 15 \mu \mathrm{A}\right)$.

Toutefois, le but de l'étude est de concevoir un déchargeur efficace certes, mais aussi non perturbant sur le plan radioélectrique. Or cette deuxième condi- 


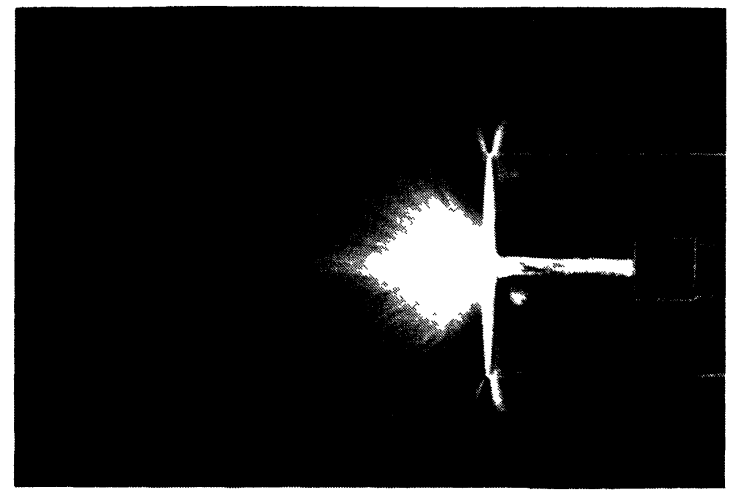

Fig. 15. - Décharges rampantes à l'intérieur du canal se prolongeant par des streamers.

[Gliding spark inside the channel prelongated by streamers.]

tion est incompatible avec la présence de décharges rampantes très énergétiques à l'intérieur du canal d'éjection. On constate expérimentalement que ces décharges apparaissent quel que soit le courant $I_{\mathrm{p}}$ à l'entrée du canal (la fréquence de déclenchement des décharges diminue lorsque $I_{p}$ décroît, mais il n'existe pas de seuil en deçà duquel elles disparaissent), ce qui laisse supposer que de telles décharges rampantes sont déclenchées par l'accumulation progressive des charges électriques sur la surface interne du canal d'éjection. Le canal percé dans le carénage électrique a donc été remplacé par un manchon de matériau à forte résistivité. Sa résistance totale est de l'ordre de $10^{10} \Omega$. Elle permet d'acheminer vers la tuyère les charges qui, à l'intérieur du manchon, s'échappent radialement de l'écoulement (Figs. 16a et $b$ ). Dans ces conditions, les décharges rampantes sont effectivement supprimées et le courant maximal éjecté se situe aux alentours de $60 \mu \mathrm{A}$ dans les deux polarités. Encore une fois, la présence d'un paramètre optimal traduisant la dualité de deux processus se manifeste. Si la résistance électrique du manchon guidant l'écoulement est trop importante, les décharges rampantes signalées plus haut apparaissent aux courants élevés. $\mathrm{Si}$, au contraire, la résistance du manchon est insuffisante, la chute ohmique du potentiel, le long du manchon sera elle aussi insuffisante ; une décharge en couronne s'allume alors à la sortie $\mathrm{du}$ jet, entretenue par la différence de potentiel entre l'extrémité du manchon et l'espace environnant, d'où la neutralisation partielle du jet chargé. Avec un manchon métallique, on retrouve le comportement du déchargeur en jet libre et le courant éjecté ne dépasse pas $6 \mu \mathrm{A}$.

6. Conclusion. - L'étude expérimentale qui vient d'être présentée montre manifestement la faisabilité d'un déchargeur à condensation d'air humide.

Bien que certains aspects du problème n'aient pu être approfondis, on peut penser que les phénomènes qui régissent le fonctionnement de ce type d'appareil ont été compris dans leurs grandes lignes.

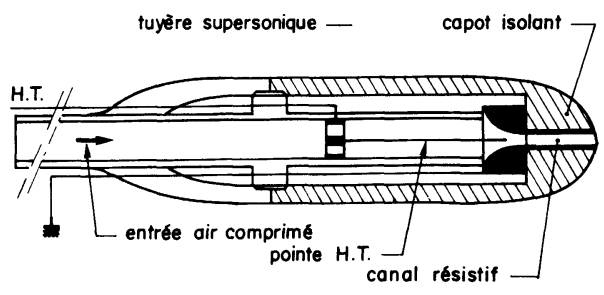

a)

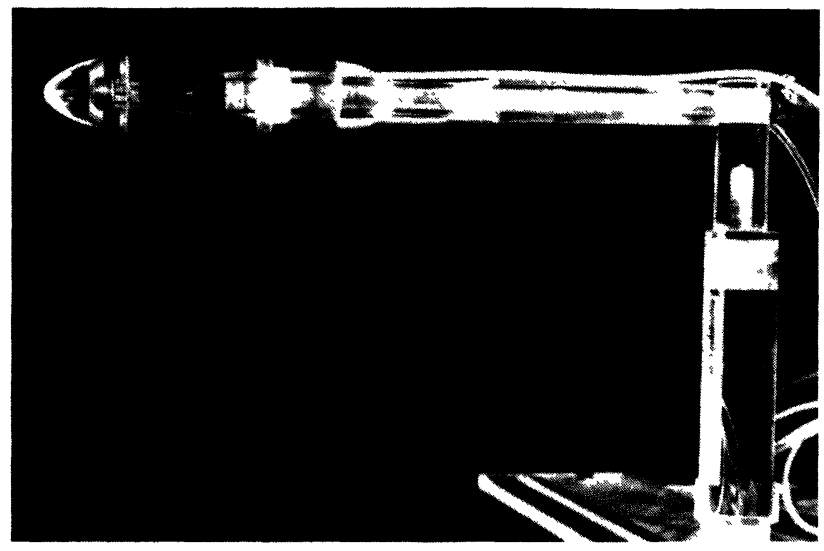

b)

Fig. 16. - Déperditeur actif.

[Active discharger.]

La mise au point d'un système d'asservissement du déchargeur au potentiel de l'hélicoptère reste évidemment à réaliser. Il s'agit là d'une étape ultérieure qui nécessitera sans doute une connaissance plus précise de l'appareil.

Dans une optique quelque peu différente, il convient de mentionner l'intérêt pratique du déchargeur pour simuler au sol les perturbations radio-électriques résultant de la charge d'un aéronef en vol. En dirigeant le jet chargé sur diverses parties de l'aéronef, on reproduit approximativement l'influence de l'effet tribo-électrique sur les verrières, les antennes, les peintures isolantes, etc... On peut ainsi examiner l'efficacité des protections électrostatiques (ou la nécessité de ces protections) directement sur l'avion ou l'hélicoptère équipé de ses instruments de radionavigation [12].

En ce qui concerne le problème général des générateurs électrofluides dynamiques, l'utilisation conjointe de particules chargées à faible mobilité et d'un canal de conversion long et étroit - dont la résistance électrique est judicieusement répartie présente un double avantage :

- possibilité de contrôle des paramètres aérodynamiques de l'écoulement, tout au long de l'espace de conversion,

- égalisation du champ axial retardateur entre l'éjecteur et le collecteur, ce qui, en retardant l'apparition du champ disruptif dans l'espace interélectrode, autorise un potentiel maximal au niveau du collecteur. 


\section{Bibliographie}

[1] Solak, B. J., L'influence de la foudre et de l'électricité statique sur la conception des hélicoptères. Traduction CEDOCAR no 1877 (Mémoire du symposium sur la foudre et l'électrostatique), San Diégo, 9-11 décembre 1970.

[2] Nanevicz, J. E., Douglas, D. G., Electrostatic discharge system. Final Report, November 1972. SRI, Menlo Park, California 94025, USA.

[3] Buser, R. G., Kauzimger, H., Inslerman, H., Dissipation des charges électrostatiques des hélicoptères gros porteurs par un système à aérosols. (Mémoire AFAL TR-72-325 Proceedings of the Lightning and Static Electricity Conference), Las Vegas, 12-15 December 1972, pp. 622-633.

[4] Whewell, B. R., BRIGHT, A. W., MAKIN, B., The application of charged aerosol to the discharge of static from aircraft. 1st International Conference on Static Electricity, Vienne (Autriche), 1970.

[5] Whewell, B. R., MaKIN, B., A study of charged condensation droplets from an aircraft discharger. Static Electricity
1971, Proceedings of the UK Institute of Physics Third Conference on Static Electrification, London, May 1971.

[6] Marks, A., Barreto, E., Chu, C. J., Charged aerosol energy converter. AIAA J. 2 (1964) no 1, p. 45-51.

[7] FéliCi, N. J., Rapport d'essais effectués sur des déperditeurs actifs. Non publié.

[8] Hermstein, W., Die stromfaden-entladung und ihr Ubergang in das Glimmen. Archiv fur Elektrotechnik (1960).

[9] KaHN, B., Gourdine, M., Electrodynamic power generation AIAA J. 2 (1964) no 8

[10] BARreto, E., Electrical discharges from and between clouds of charged aerosols. J. Geophys. Res. 74 (1969) no 28.

[11] Barreto, E., Martinot, K. O., Nonthermal ionisation caused by aerodynamic discontinuities in charged aerosols jets. Phys. Fluids 10 (1967), no 10, pp. 2155-2163.

[12] TAILLET, J., Method of assessment of the antistatic protection of aircraft. Conference on Electrostatic, Oxford, 1820/04/1979. 